\title{
Fluorescence lifetime components reveal kinetic intermediate states upon equilibrium denaturation of carbonic anhydrase II
}

\author{
Elena V. Nemtseva $\$$, Olesya O. Lashchuk $\$$, Marina A. Gerasimova $\ddagger$, Tatiana N. Melnik $\uparrow$, Galina S. \\ Nagibina $\uparrow$, Bogdan S. Melnik*† \\ ¥Siberian Federal University, 660041 Krasnoyarsk, Russia \\ §Institute of Biophysics SB RAS, Federal Research Center 'Krasnoyarsk Science Center SB RAS', \\ 660036, Krasnoyarsk, Russia
}

$\dagger$ Institute of Protein Research, Russian Academy of Sciences, 142290 Pushchino, Moscow Region, Russia

${ }^{1}$ Abbreviations: BCA II, Bovine carbonic anhydrase II

\begin{abstract}
In most cases, intermediate states of multistage folding proteins are not "visible" under equilibrium conditions but are revealed in kinetic experiments. Time-resolved fluorescence spectroscopy was used in equilibrium denaturation studies. The technique allows detecting changes in the conformation and environment of tryptophan residues in different structural elements of carbonic anhydrase II which in its turn has made it possible to study intermediate states of carbonic anhydrase II under equilibrium conditions. The results of equilibrium and kinetic experiments using wild-type bovine carbonic anhydrase II and its mutant form with the substitution of leucine for alanine at position 139 (L139A) were compared. The obtained lifetime components of intrinsic tryptophan fluorescence allowed revealing that the same as in kinetic experiments, under equilibrium conditions the unfolding of carbonic anhydrase II ensues through formation of intermediate states.
\end{abstract}

KEYWORDS: carbonic anhydrase II, time-resolved fluorescence spectroscopy, protein intermediate states, comparing of kinetic and equilibrium experiments, protein fluorescence lifetime

\section{Introduction}

Bovine carbonic anhydrase II (BCA II) is a suitable model protein for studying peculiarities of formation/breakdown of the structure of large single-domain proteins. This protein is readily isolated and can be renatured in vitro with its function being restored. ${ }^{1}$ Formation of the BCA II native structure is relatively slow: it takes from several minutes under native conditions to several hours in the presence of denaturing agents. ${ }^{1-3}$ Slow folding and unfolding of carbonic anhydrase II allow performing simple kinetic experiments with manual mixing of the protein with denaturant. As a result, both the folding of this protein and peculiarities of its intermediate states have been thoroughly studied with the use of different physicochemical methods. For example, the stability and formation of BCA II intermediate states were analyzed using circular dichroism, fluorescence, DSC, absorption and mass spectrometry (the slow $\mathrm{H} / \mathrm{D}$ exchange reaction) methods. ${ }^{1-4,5}$

But notwithstanding the great number of studies devoted to carbonic anhydrase II, it has remained unclear whether for this protein the same conditions are observed in kinetic and equilibrium experiments. This problem is also urgent for many other proteins. Under equilibrium conditions only the most stable states are populated in proteins and therefore they can be observed. In kinetic experiments, the states separated from others by a large energy barrier are mostly "visible"; but these are not the most stable states. Thus it can appear that for a multistage folding protein some intermediate states can be "visible" only under equilibrium conditions while the others only in kinetic experiments.

If BCA II is studied under equilibrium conditions by the methods of circular dichroism and steadystate tryptophan fluorescence it may seem that folding/unfolding of this protein occurs in one stage. In any case, it is rather difficult to reveal and characterize at least one intermediate state. Meanwhile, kinetic experiments demonstrate that folding and unfolding of this protein occurs at least in three stages via formation of two intermediate states. ${ }^{1-4,6}$ Previous studies of carbonic anhydrase II showed that one of the intermediate states is quite structured while the other is the molten globule state. ${ }^{2}$ Thus, one of these states is very similar to the native state by its optical properties (tryptophan fluorescence and ellipticity) and the other is similar to the denatured state. Therefore, the results of equilibrium experiments can be used to plot only one S-like curve, which would reflect the averaged protein transition from structured states to 
states with disrupted structure. Experimentalists face such challenge rather frequently. For example, under equilibrium conditions lysozyme, apomyoglobin, ribonuclease A, barnase and some other proteins behave as two-state proteins, and kinetic experiments allow revealing intermediate states formed upon their folding. ${ }^{7-13}$ The solution of this problem is to find an experimental method that is sensitive enough to distinguish between different intermediate states of the protein. In this study we have demonstrated that for carbonic anhydrase II the stability of intermediate states upon denaturation can be recorded experimentally by the change in the lifetime components of intrinsic tryptophan fluorescence of this protein.

\section{MATERIALS AND METHODS}

Protein expression and purification. The bovine carbonic anhydrase II coding sequence was reverse-transcribed from total RNA of bone marrow cells. cDNA was synthesized using gene specific oligonucleotide primer 3`-CA2 (5`-tttgtcgacGGCCAGTTCACCAAGTGGACTTGTG-3` (SalI restriction site is underlined) and M-MuLV reverse transcriptase (Fermentas, Lithuania). Products of first strand synthesis were amplified using the polymerase chain reaction and gene specific oligonucleotide primer 5'-CA2 (5'-tacttttcatATGTCCCATCACTGGGGATAC-3') (NdeI restriction site is underlined). The amplified carbonic anhydrase II gene was double digested with SalI and NdeI and inserted into the pET11c_joe vector between NdeI and SalI restriction sites. The resulting plasmid was designated as pBCAB. Plasmids with the mutant BCA II genes were constructed by a standard PCR technique, using appropriate primers and a pET-28a vector as a template and a QuikChange kit (Stratagene, USA). The DNA sequences of all constructs were confirmed by the DNA sequence analysis. Carbonic anhydrase II and its mutant forms were expressed in $E$. coli cells and isolated as described elsewhere. ${ }^{14,15}$

Unfolding and refolding experiments. For refolding studies, samples of unfolded carbonic anhydrase II (solution containing $9.5 \mathrm{M}$ urea, $20 \mathrm{mM}$ phosphate buffer, $\mathrm{pH}$ 8) were prepared at high protein concentrations (usually $2-10 \mathrm{mg} / \mathrm{ml}$ ) and incubated in $9.5 \mathrm{M}$ urea for $24 \mathrm{~h}$ in order to reach complete unfolding. Refolding experiments were initiated by 10- to 100 -fold dilution of the unfolded protein into the phosphate buffer $(20 \mathrm{mM}$ phosphate buffer, $\mathrm{pH} 8)$ containing desired urea concentrations. The final protein content in the refolding mixtures varied from 0.01 to $1.0 \mathrm{mg} / \mathrm{ml}$. Experiments on the carbonic anhydrase II unfolding were carried out in a similar manner, by 10 - to 100 -fold dilution of the native protein to solutions containing desired urea concentrations in $20 \mathrm{mM}$ phosphate buffer, $\mathrm{pH} 8$. Fluorescence unfolding and refolding kinetics were monitored using a Cary Varian Eclipse spectrofluorometer (Agilent Technologies, Australia) equipped with a temperature-controlled holder. The samples were excited at $280 \mathrm{~nm}$ and the emission was monitored at $335 \mathrm{~nm}$ using the cuvette with $10 \times 10$ $\mathrm{mm}$ path lengths. Slits of $2 \mathrm{~nm}$ were applied for excitation and emission. All measurements were performed in $20 \mathrm{mM}$ phosphate buffer at $\mathrm{pH} 8,20^{\circ} \mathrm{C}$. All obtained kinetic curves were well fitted by a double-exponential approximation. Kinetic data were repeatedly obtained five times for each point. Kinetic experiments were performed in the absence of zinc which allowed excluding the slow phase of zinc binding detected only upon measuring the kinetics of folding.

Steady-state and time-resolved fluorescence measurements. Steady-state and time-resolved fluorescence measurements were performed using Fluorolog 3-22 spectrofluorometer (Horiba Jobin Yvon, USA) equipped with DeltaHub timing module for the time-correlated single photon counting. For steady-state experiments $450 \mathrm{~W}$ Xenon arc lamp was used as an excitation source, for time-resolved NanoLED pulsed diode (296 nm, a pulse duration of $\sim 1.2 \mathrm{~ns}$ ) was applied. Steady-state fluorescence spectra were corrected for the background intensities and for the inner filter effect. Time-resolved fluorescence decays were collected in the range of 305-419 nm with increment of $3 \mathrm{~nm}$ and a time resolution of $27 \mathrm{ps} / \mathrm{channel}$.

The UV-Vis absorption spectra were measured with a Cary 5000i spectrophotometer (Agilent Technologies, Australia). The final protein concentration $(6.6 \mu \mathrm{M})$ was calculated from absorption at 280 $\mathrm{nm}$ using the extinction coefficient $54.5 \cdot 10^{3} \mathrm{M}^{-1} \mathrm{~cm}^{-1}$. Equilibrium denaturation was performed by incubating the proteins during not less than 12 hours at $20^{\circ} \mathrm{C}$ in $20 \mathrm{mM}$ Tris- $\mathrm{HCl}$ buffer, $\mathrm{pH} 8.2$, containing increasing concentrations of urea $(0-7.5 \mathrm{M})$. The final concentration of urea was defined from refractive index measured by IRF-454-B2 M (KOMZ, Kazan, Russia). 
In order to recover the fluorescence decay parameters (amplitudes and lifetimes), the global analysis approach ${ }^{16}$ was carried out using deconvolution DAS6 software (Horiba). The set of 39 decays collected for each sample was fitted with one, two, three and four lifetimes. The fit quality was evaluated by its global $\chi^{2}$ value and weighted residuals plot. It was found that for all samples the model with three exponential components gives the best fitting of the experimental data. As a result, the time-resolved fluorescence decay at the wavelength $\lambda$ was described as a sum of three exponents:

$$
I_{\lambda}(t)=\sum_{i=1}^{3} \alpha_{i}^{\lambda} \exp \left(-t / \tau_{i}\right)
$$

where $\tau_{i}$ is the lifetime and $\alpha_{i}^{\lambda}$ is the amplitude of $i$-component. The fractional fluorescence intensity $f_{i}^{\lambda}$ of each lifetime component was determined as

$$
f_{i}^{\lambda}=\frac{\alpha_{i}^{\lambda} \tau_{i}}{\sum_{i} \alpha_{i}^{\lambda} \tau_{i}} .
$$

Then normalized contribution of $i$-component to the steady-state fluorescence intensity $A_{i}$ was calculated as

$$
A_{i}=\frac{\sum_{\lambda} I_{s s}^{\lambda} f_{i}^{\lambda}}{\sum_{\lambda} I_{s s}^{\lambda}},
$$

where $I_{s s}^{\lambda}$ is the steady-state fluorescence intensity at wavelength $\lambda$. software.

The results were processed and analyzed using Microsoft Excel and Microcal Origin Pro 8.1

\section{RESULTS AND DISCUSSION}

Equilibrium and kinetic studies of carbonic anhydrase II by steady-state fluorescence techniques.

The study of bovine carbonic anhydrase II denaturation under equilibrium conditions by means of steady-state intrinsic fluorescence can lead to an erroneous conclusion about the number of stages upon folding/unfolding of this protein. For example, Figure 1 shows equilibrium unfolding curves of BCA II plotted using the following common experimental parameters: intensity of steady-state fluorescence at fixed wavelength, e.g. $335 \mathrm{~nm},\left(\mathrm{I}_{335}\right)$ and fluorescence spectrum shift $\left(\mathrm{I}_{320} / \mathrm{I}_{380}\right)$. As seen in figure, the curves are compatible with two-state protein unfolding model for carbonic anhydrase II with the transition midpoint at $6.2 \pm 0.1$.

However kinetic experiments reveal two slow steps of BCA II folding and unfolding. ${ }^{2}$ From the kinetic curves followed by steady-state fluorescence intensity two rate constants for both folding and unfolding reaction of this protein can be calculated. ${ }^{1-3}$ In addition, the amplitude change in the kinetic curves upon folding allows finding the fast step of formation of intermediate state similar to molten globule. ${ }^{2}$ Figure 2 shows a chevron plot (dependency of the logarithm of folding and unfolding rates on the urea concentration) formed for BCA II from kinetic measurements of fluorescence intensity change at the wavelength $335 \mathrm{~nm}$. A detailed description of the kinetic curves, their analysis and the method for calculating rate constants have been given elsewhere ${ }^{2,7}$. It was demonstrated in Ref. 2 that the kinetic data obtained by the method of fluorescence are compatible with ones obtained by the circular dichroism and absorption techniques. As a result of the kinetic data analysis we can describe the process of carbonic anhydrase II folding/unfolding by the following scheme. ${ }^{2}$

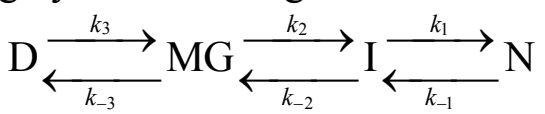

Here $\mathrm{D}$ is the denatured state; $\mathrm{MG}$ is the first intermediate state similar to molten globule; $\mathrm{I}$ is the second intermediate state; $\mathrm{N}$ is the native state.

The kinetic data on carbonic anhydrase II folding/unfolding were used to calculate the rate constants for transitions between states MG, I and N. In Figure 2, the rate constants refer to the transition between BCA II states in accordance with the scheme (1). Approximation of the complicated chevron 
plot requires a special explanation and mathematical calculations as described in detail previously., ${ }^{2,7,17}$ For the current purposes, it is sufficient to have a general form of the chevron plot obtained from experiment without its mathematical analysis. However, it is noteworthy that the minimum of the chevron plot corresponds to the concentration of the denaturing agent when the folding of the protein turns into its unfolding. Thus, if in kinetic and equilibrium experiments we study the same states of the protein, the position of the minimum of the chevron plot should coincide with the midpoint of the sigmoid curve of equilibrium transition of the protein. ${ }^{18}$

Comparison of Figures 1 and 2 shows that it is difficult to juxtapose the position of two minima of chevron plots for wild-type BCA II with the midpoint of the transition curve obtained for this protein under equilibrium conditions. It is not clear whether the equilibrium curve reflects either the disruption of one state of BCA II or the "averaged" unfolding of several states. Thus, from steady-state fluorescence data obtained in equilibrium experiments it is impossible to reveal intermediate states of carbonic anhydrase II which are observed in kinetic experiments.

In some cases, such divergence of kinetic and equilibrium experiments is explained by a simple statement that "kinetic intermediate states" are not the same as "equilibrium intermediate states". If this is the case, whatever method we choose, we will always have diverging results of kinetic and equilibrium experiments. On the other hand, if intermediate states of the protein in kinetic and equilibrium experiments are the same, it means that the only problem is to find a proper experimental approach that allows distinguishing different intermediate states of the protein under equilibrium conditions.

Is it possible to choose an experimental method that would allow distinguishing different intermediate states of carbonic anhydrase II under equilibrium conditions?

As previously shown, ${ }^{5}$ special stages of carbonic anhydrase II unfolding are related to destruction of different structural elements of this protein. We assumed that it is possible to distinguish between special states of BCA II under equilibrium conditions using the method which allows observing selectively different structural parts of the protein. In the case of proteins, containing tryptophans in their structure, just these residues can be a suitable internal labels reporting about structural changes in the particular protein domain. An experimental method, which may allow differentiating tryptophan residues in different microenvironments, is time-resolved fluorescence spectroscopy.

\section{Equilibrium studies of carbonic anhydrase II unfolding by time-resolved fluorescence of protein}

On the one hand, time-resolved characteristics of fluorescence can provide additional useful information on the structure of fluorophore and its interactions with the surrounding like energy transfer, dynamic quenching, excited-state reactions and other processes. ${ }^{19}$ On the other hand, the analysis of the protein fluorescence lifetimes faces some challenges because the tryptophan fluorescence decay is not monoexponential both in solution and within proteins. In most cases it can be described by two or three components. ${ }^{19,20}$ Moreover, in spite of the structure distinctions the fluorescence decays measured for different proteins can be similar and featured by three characteristic lifetimes of about $0.1-0.5,1-3$ and $4-7$ ns. $^{21-23}$

We have measured tryptophan fluorescence decays of wild-type carbonic anhydrase II in solution with different concentrations of urea $(0-7.5 \mathrm{M})$. Global analysis of 39 fluorescence decays for each sample revealed that this set is fitted by the sum of three exponents with characteristic times $\tau_{1}=5.0-5.6$ ns, $\tau_{2}=1.1-2.0 \mathrm{~ns}$ and $\tau_{3}=0.14-0.23 \mathrm{~ns}$. It was obtained that in the native state protein fluorescence is determined mainly by the long lifetime component $\tau_{1}$ (its spectral contribution is about $84 \%$ ); in the denatured state this contribution decreases to $55 \%$. The contribution of the middle lifetime component $\tau_{2}$ to steady-state spectra increases from $9.4 \%$ to $34 \%$ as a result of unfolding. The values of the individual lifetime component and its contribution into total steady-state fluorescence of the sample alter in a complicated way, depending on the concentration of urea in solution, so reflecting structural transitions of the protein. It is noteworthy that the change in the short lifetime component $\left(\tau_{3} \leq 0.23 \mathrm{~ns}\right)$ could not be reliably measured due to limited instrument resolution. But its contribution to fluorescence of the samples did not exceed $8 \%$ which was the reason for excluding it from our consideration for a while.

Figures $3 \mathrm{~A}$ and $3 \mathrm{~B}$ show dependencies of the fluorescence lifetime components $\left(\tau_{1}\right.$ and $\left.\tau_{2}\right)$ of the wild-type BCA II versus the urea concentration in solution. As seen from Figures $3 \mathrm{~A}$ and $3 \mathrm{~B}$, the values of both fluorescence lifetimes also shift upon unfolding and demonstrate transition in the range between 5 
and 6.5 $\mathrm{M}$ urea. The transition midpoints for $\tau_{1}$ and $\tau_{2}$ dependencies were found to be $6.4 \pm 0.1 \mathrm{M}$ and $5.7 \pm 0.1 \mathrm{M}$, respectively. These midpoints are in good agreement with ones of chevron plots, i.e. with the concentrations of urea when protein folding turns to be unfolding in kinetic experiments (Figure 2). In Figures 2 and 3, arrows indicate the minimums of chevron plots and the midpoints of equilibrium transitions.

In Figure $3 \mathrm{C}$ the normalized contributions to the steady-state fluorescence corresponding to $\tau_{1}$ and $\tau_{2}, A_{1}$ and $A_{2}$, are depicted. The dependencies of $A_{1}$ and $A_{2}$ on the urea concentration for wild-type BCA II reveal the same patterns: the transition starts at about $5 \mathrm{M}$ urea and terminates at about $6.4 \mathrm{M}$ with the midpoint at $5.78 \pm 0.05 \mathrm{M}$. It implies that the dependencies are mainly guided by fraction redistribution of two species with negligible influence from the third component, $A_{3}$. The close position of midpoints for $\tau_{2}$ and $A_{l}\left(A_{2}\right), 5.7 \pm 0.1$ versus $5.78 \pm 0.05 \mathrm{M}$, can be result of more pronounced change of $\tau_{2}$ in comparison with $\tau_{1}$ (Figure $3 \mathrm{~A}, \mathrm{~B}, \mathrm{C}$ ). Under denaturation the middle lifetime $\tau_{2}$ increases up to 1.6-fold, whereas long lifetime $\tau_{1}$ shortens only up to 1.1 -fold. The increasing lifetime $\tau_{2}$ causes the enhanced intensity and higher contribution, $A_{2}$, into steady-state spectra. Thus, the transition curves for $A_{1}$ and $A_{2}$ bring nearly the same information as $\tau_{2}$ shift.

It should be acknowledged that folding/unfolding processes of carbonic anhydrase II wild-type take place in a rather narrow range of urea concentrations. This may be the reason for uncertainties whether the obtained coincidence between the kinetic data and the results of time-resolved protein fluorescence is accidental. To corroborate the obtained results the BCA II mutant was analyzed using precisely the same experimental techniques and procedures.

The amino acid residue L139 located on the $\beta$-sheet in the center of carbonic anhydrase II (PDB:1v9e) was chosen for substitution. In our opinion, mutation in this position should destabilize not only the native state of BCA II but also its intermediate state. At the same time, judging by steady-state fluorescence spectra, the structures of both the native and completely denatured state of L139A mutant do not differ from those for wild-type protein.

Data obtained for the mutant form of BCA II with the substitution of L139A are shown by empty symbols in Figures 1-3. Similarly to the case of wild type protein, equilibrium studies with the use of steady-state fluorescence do not yield information on the intermediate state of L139A mutant (Figure 1). Transition curves look like those for two-state protein with the midpoint at $4.59 \pm 0.01 \mathrm{M}$. But the kinetic curves of L139A mutant folding and unfolding allow calculating two rate constants and constructing a chevron plot (Figure 2). Figure 2 shows that the branches of folding in the chevron plot for the mutant protein coincide with the branches of folding for wild-type protein (from 0 to $4 \mathrm{M}$ urea), while the branches of unfolding shifted upwards significantly. This means that mutation L139A has accelerated two stages of carbonic anhydrase II unfolding. Such changes in chevron plots appear when the amino acid substitution destabilizes the native and intermediate states of the protein but does not affect its activated (transient) states. ${ }^{6,24}$

The dependencies of $\tau_{1}$ and $\tau_{2}$ on the urea concentration for L139A mutant were found to reveal similar information on kinetic data (Figure 3A, B). The transition curves of mutant protein are shifted to lower urea concentration indicating that substitution L139A has destabilized the native and intermediate states BCA II. The midpoints of equilibrium transitions were determined as $5.00 \pm 0.03 \mathrm{M}$ and $4.22 \pm 0.04$ $\mathrm{M}$ for $\tau_{1}$ and $\tau_{2}$ dependencies, respectively. And again, found midpoints for mutant protein L139A (Figures 3A, B) are compatible with the minimums of its chevron plot (Figure 2). The transition curves of spectral contributions $A_{1}$ and $A_{2}$ for L139A have the midpoints at $4.40 \pm 0.05 \mathrm{M}$ (Figure 3C) that is close to the characteristic of curve for $\tau_{2}$ (Figure $3 \mathrm{~B}$ ).

\section{The origin of changes in fluorescence lifetimes of carbonic anhydrase II}

The interpretation of spectral parameters of carbonic anhydrase II intrinsic fluorescence is not a trivial task since this protein contains 7 tryptophan residues in positions 4, 15, 96, 122, 190, 207 and 243. Thus, the observed fluorescence characteristics are superposition of that from several emitters. To simplify the consideration we grouped the BCA II tryptophan residues in accordance with microenvironment features that cause the spectral properties. A web-based toolkit PFAST was used for classification of tryptophan residues in carbonic anhydrase II structure (http://pfast.phys.uri.edu ${ }^{25}$ ). 
Structural-correlation analysis tools, SCAT, assigned every tryptophan residue to one of five spectralstructural classes relying on the crystal structure of the protein (PDB: 1V9E). The detailed description of five tryptophan classes can be found elsewhere. ${ }^{26}$ The result of structural analysis is shown in Table 1.

It was found that in folded state three tryptophan residues of BCA II (W15, W96 and W207) belong to class S and one (W190) - to class I. It means that they are deeply buried inside protein and have free partners for hydrogen bond formation nearby. The remaining three tryptophans could be assigned to two classes (W122 - to S or I, W4 and W243 - to I or II). Class II that is probable for the tryptophans located on the chain ends (W4 and W243) implies the surface location and presence of the structured-water molecule near to indole ring. This classification of BCA II tryptophans is in good agreement with the data obtained earlier for its close homologue - human carbonic anhydrase. ${ }^{26}$

The theory of discrete spectral classes of tryptophan residues in proteins has been developed relying on steady-state fluorescence of the proteins. ${ }^{27}$ But inclusion of time-resolved properties of polypeptides into this classification currently remains challenging. Even single-tryptophan proteins that belong with high probability to a particular spectral class display two or three fluorescence lifetime components. ${ }^{21,22}$ The common explanation for multiple fluorescence lifetimes of tryptophan is the existence of its rotamers (conformational isomers) both in solution and within proteins. ${ }^{28}$ Taking this idea into account we can propose that belonging to particular spectral-structural class causes the formation of tryptophan rotamers with particular relative probability. In turn, the interaction with microenvironment modulates the value of each lifetime. Interestingly, the buried tryptophan residues were found to display shorter lifetimes, probably due to quenching by peptide bonds. ${ }^{19}$ In our case, the steady-state fluorescence of native BCA II is determined by the component with long lifetime $\tau_{1}\left(\mathrm{~A}_{1}\right.$ is about $\left.80 \%\right)$ that means significant contribution from the exposed tryptophan residues (class II). It is confirmed by the spectral peak of the steady-state fluorescence that is at about $337 \mathrm{~nm}$. Thus we can assume that the shift in $\tau_{1}$ displays the change in W4 and W243 microenvironment, whereas $\tau_{2}$ variations originate from the structural change around buried tryptophan residues W15, W96 and W207.

The compatibility of the kinetic data with the data obtained under equilibrium conditions (Figures 2,3 ) makes it possible to conclude that under equilibrium and non-equilibrium conditions the formation of native structure of carbonic anhydrase II occurs via formation of similar intermediate states. The method of time-resolved fluorescence spectroscopy allows observing the process of formation and destruction of intermediate states of BCA II under equilibrium conditions.

\section{CONCLUSIONS}

When carbonic anhydrase II is studied using the method of fluorescence or circular dichrosim under equilibrium conditions at different concentrations of the denaturing agent, it is possible to plot an S-like curve of transition that is characteristic of two-state proteins. This curve does not allow concluding on the accumulation of intermediate states. But when such studies are performed using time-resolved fluorescence spectroscopy and the fluorescence lifetimes are analyzed, it can be found that unfolding of carbonic anhydrase II occurs via formation of intermediate states. The compatibility of equilibrium transitions plotted using the fluorescence lifetimes $\tau_{1}$ and $\tau_{2}$ with kinetic data shows that these parameters make it possible to observe the destruction of the intermediate and native states of carbonic anhydrase II. The results of studies obtained both for wild-type carbonic anhydrase II and its destabilized mutant with the substitution of L139A demonstrate that "kinetic" and "equilibrium" intermediate states of carbonic anhydrase II have the same stability (the midpoint of chevron plots coincides with the midpoint of equilibrium transitions). Thus, it can be concluded that in kinetic and equilibrium experiments the folding/unfolding of carbonic anhydrase II occurs via formation of similar intermediate states.

For proteins, containing tryptophan residues, the fluorescence lifetime seems to be a promising parameter to observe folding/unfolding of different intermediate states of a protein under equilibrium conditions.

\section{ACKNOWLEDGMENT}

The study of time-resolved protein fluorescence was supported by the Ministry for Science and Education of the Russian Federation (project 6.7734.2017/BCH). Kinetic and genetic engineering studies of carbonic anhydrase II were supported by grant N14-24-00157 from the Russian Science Foundation. 
Table 1. Probabilities that the tryptophan residue of BCA II belongs to a particular spectral class ${ }^{26}$ obtained by $\mathrm{SCAT}^{25}$

\begin{tabular}{|l|l|l|l|l|l|}
\hline \multirow{2}{*}{ Residue } & \multicolumn{5}{|c|}{ Probabilities } \\
\cline { 2 - 6 } & Class A & Class S & Class I & Class II & Class III \\
\hline W4 & 0 & 0.02 & $\mathbf{0 . 6 3}$ & $\mathbf{0 . 3 5}$ & 0 \\
\hline W 15 & 0.005 & $\mathbf{0 . 9 3 5}$ & 0.06 & 0 & 0 \\
\hline W 96 & 0.02 & $\mathbf{0 . 9 6}$ & 0.02 & 0 & 0 \\
\hline W 122 & 0 & $\mathbf{0 . 4 1 8}$ & $\mathbf{0 . 5 7 7}$ & 0.005 & 0 \\
\hline W 190 & 0 & 0.14 & $\mathbf{0 . 8 2}$ & 0.04 & 0 \\
\hline W 207 & 0.027 & $\mathbf{0 . 9 5 5}$ & 0.018 & 0 & 0 \\
\hline W 243 & 0 & 0.033 & $\mathbf{0 . 6 8 8}$ & $\mathbf{0 . 2 7 9}$ & 0 \\
\hline
\end{tabular}

\section{FIGURES}

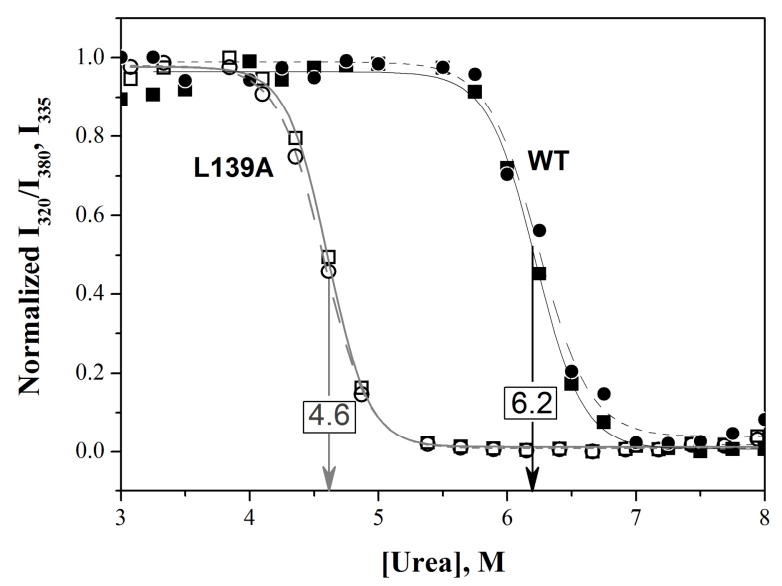

Figure 1. Equilibrium unfolding of carbonic anhydrase II wild type (WT) (filled symbols) and its mutant L139A (empty symbols) detected by fluorescence intensity at $335 \mathrm{~nm}$ (squares) and shift of the fluorescence spectra $\mathrm{I}_{320} / \mathrm{I}_{380}$ (circles). Solid line curves refer to the fitting by the sigmoid function. Arrows with numbers indicate the urea concentrations corresponding to transition midpoints.

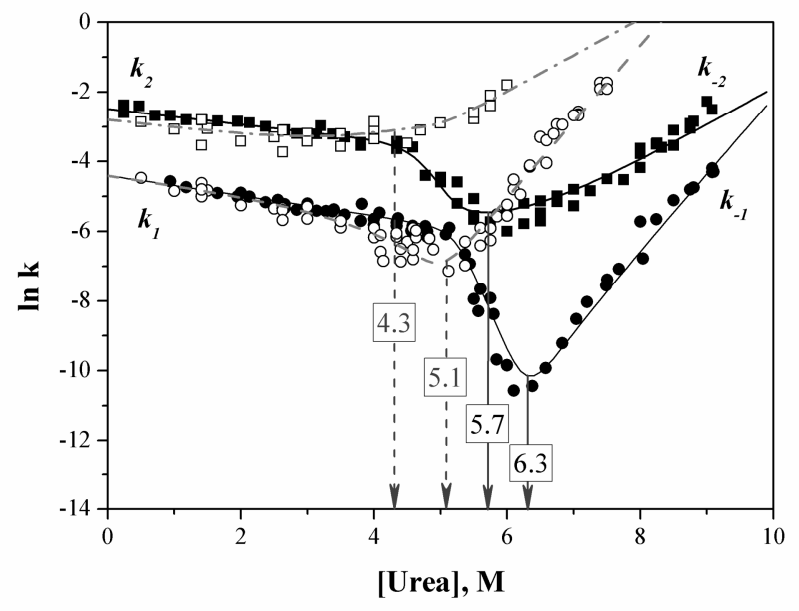

Figure 2. Chevron plots for carbonic anhydrase II wild type (filled symbols) and its mutant L139A (empty symbols). Circles show the slow phase of folding/unfolding reflecting protein transition from native $(\mathrm{N})$ to intermediate state (I). Squares refer to the transition rates from intermediate state (I) to molten globule state (MG). Arrows with numbers indicate urea concentrations at which protein folding turns into unfolding. Peculiarities of approximation of chevron plots are described elsewhere. ${ }^{2}$ 
A

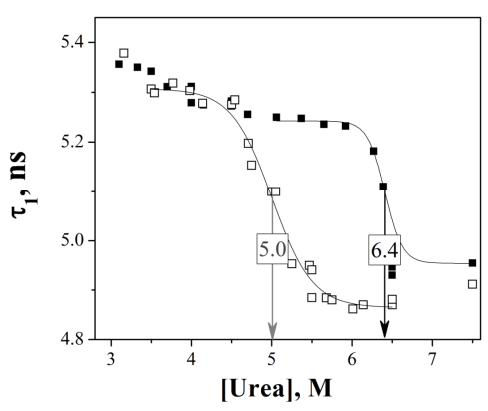

B

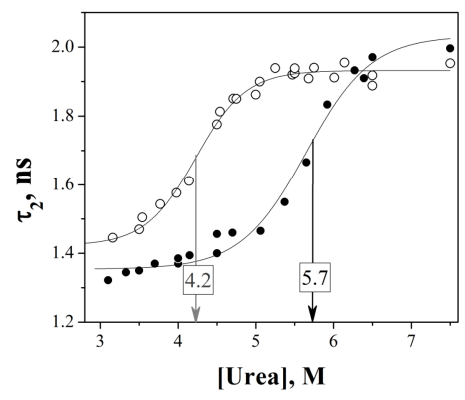

C

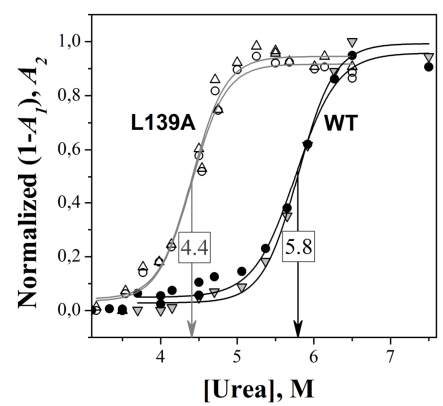

Figure 3. Dependencies of fluorescence lifetimes (A and B) and their contributions (C) on urea concentration for carbonic anhydrase II wild type (filled symbols) and its mutant L139A (empty symbols). A, Longer lifetime component $\tau_{1}$ associated mainly with exposed tryptophan residues. B, Middle lifetime component $\tau_{2}$ associated mainly with buried tryptophan residues. C, Normalized contributions $A_{1}$ and $A_{2}$ to the steady-state fluorescence spectra corresponding to the components $\tau_{1}$ and $\tau_{2}$ (triangles and circles, correspondently). Solid line curves refer to the fitting by sigmoid function. Arrows with numbers indicate the urea concentrations corresponding to transition midpoints.

\section{REFERENCES}

(1) Semisotnov, G. V., Uversky, V. N., Sokolovsky, I. V., Gutin, A. M., Razgulyaev, O. I., and Rodionova, N. A. (1990) Two slow stages in refolding of bovine carbonic anhydrase B are due to proline isomerization. J. Mol. Biol. 213, 561-568.

(2) Melnik, B. S., Marchenkov, V. V, Evdokimov, S. R., Samatova, E. N., and Kotova, N. V. (2008) Multy-state protein: Determination of carbonic anhydrase free-energy landscape. Biochem. Biophys. Res. Commun. 369, 701-706.

(3) Semisotnov, G. V., Rodionova, N. A., Kutyshenko, V. P., Ebert, B., Blanck, J., and Ptitsyn, O. B. (1987) Sequential mechanism of refolding of carbonic anhydrase B. FEBS Lett. 224, 9-13.

(4) Tran, D. T., Banerjee, S., Alayash, A. I., Crumbliss, A. L., and Fitzgerald, M. C. (2012) Slow histidine H/D exchange protocol for thermodynamic analysis of protein folding and stability using mass spectrometry. Anal. Chem. 84, 1653-1660.

(5) Melnik, B. S., Nagibina, G. S., Glukhov, A. S., and Melnik, T. N. (2016) An approach for the assessment of the order of disruption of the elements of protein structure upon protein unfolding: A study of carbonic anhydrase B. Biophysics (Oxf). 61, 860-870.

(6) Melnik, B. S., Nagibina, G. S., Glukhov, A. S., Melnik, T. N., and Uversky, V. N. (2016) Substitutions of Amino Acids with Large Number of Contacts in the Native State Have no Effect on the Rates of Protein Folding. Biochim. Biophys. Acta 1864, 1809-1817.

(7) Baryshnikova, E. N., Melnik, B. S., Finkelstein, A. V, Semisotnov, G. V, and Bychkova, V. E. (2005) Three-state protein folding: experimental determination of free-energy profile. Protein Sci. 14, $2658-67$.

(8) Li, L., and Chang, J. Y. (2004) Two-state folding of lysozyme versus multiple-state folding of $\alpha$-lactalbumin illustrated by the technique of disulfide scrambling. Protein J. 23, 3-10.

(9) Bachmann, A., Segel, D., and Kiefhaber, T. (2002) Test for cooperativity in the early kinetic intermediate in lysozyme folding. Biophys. Chem. 96, 141-151.

(10) Arnold, U. (2014) Stability and folding of amphibian ribonuclease A superfamily members in comparison with mammalian homologues. FEBS J. 281, 3559-75.

(11) Garel, J. R., and Baldwin, R. L. (1973) Both the fast and slow refolding reactions of ribonuclease A yield native enzyme. Proc. Natl. Acad. Sci. U. S. A. 70, 3347-51.

(12) Vu, N.-D., Feng, H., and Bai, Y. (2004) The Folding Pathway of Barnase: The Rate-Limiting Transition State and a Hidden Intermediate under Native Conditions. Biochemistry 43, 3346-3356.

(13) Fersht, A. R. (2000) A kinetically significant intermediate in the folding of barnase. Proc. Natl. Acad. Sci. 97, 14121-14126.

(14) Dolgikh, D. A., Kolomiets, A. P., Bolotina, I. A., and Ptitsyn, O. B. (1984) "Molten-globule" 
state accumulates in carbonic anhydrase folding. FEBS Lett. 165, 88-92.

(15) Armstrong, J. M., Myers, D. V, Verpoorte, J. A., and Edsall, J. T. (1966) Purification and properties of human erythrocyte carbonic anhydrases. J.Biol.Chem. 241, 5137-5149.

(16) Beechem, J. M., Gratton, E., Ameloot, M., Knutson, J. R., and Brand, L. The Global Analysis of Fluorescence Intensity and Anisotropy Decay Data: Second-Generation Theory and Programs, in Topics in Fluorescence Spectroscopy, pp 241-305. Kluwer Academic Publishers, Boston.

(17) Samatova, E. N., Melnik, B. S., Balobanov, V. A., Katina, N. S., Dolgikh, D. A., Semisotnov, G. V, Finkelstein, A. V, and Bychkova, V. E. (2010) Folding Intermediate and Folding Nucleus for I $\rightarrow$ N and $\mathrm{U} \rightarrow \mathrm{I} \rightarrow \mathrm{N}$ Transitions in Apomyoglobin: Contributions by Conserved and Nonconserved Residues. Biophys. J. 98, 1694-1702.

(18) Finkelstein, A. V., and Ptitsyn, O. B. (2016) Protein Physics. A Course of Lectures. Acad. Press is an Impr. Elsevier 2nd Ed. Amsterdam.

(19) Lakowicz, J. R. (Ed.). (2006) Principles of Fluorescence Spectroscopy. Springer US, Boston, MA.

(20) Albani, J. R. (2014) Origin of tryptophan fluorescence lifetimes. Part 1. Fluorescence lifetimes origin of tryptophan free in solution. J. Fluoresc. 24, 93-104.

(21) Swaminathan, R., Krishnamoorthy, G., and Periasamy, N. (1994) Similarity of fluorescence lifetime distributions for single tryptophan proteins in the random coil state. Biophys. J. 67, 2013-2023.

(22) Albani, J. R. (2014) Origin of tryptophan fluorescence lifetimes. Part 2: fluorescence lifetimes origin of tryptophan in proteins. J. Fluoresc. 24, 105-117.

(23) Nemtseva, E. V., Lashchuk, O. O., and Gerasimova, M. A. (2016). Similarity of decayassociated spectra for tryptophan fluorescence of proteins with different structures. Biophysics, 61, 193199.

(24) Fersht, A. R., and Sato, S. (2004) Phi-value analysis and the nature of protein-folding transition states. Proc. Natl. Acad. Sci. U. S. A. 101, 7976-81.

(25) Shen, C., Menon, R., Das, D., Bansal, N., Nahar, N., Guduru, N., Jaegle, S., Peckham, J., and Reshetnyak, Y. K. (2008) The protein fluorescence and structural toolkit: Database and programs for the analysis of protein fluorescence and structural data. Proteins 71, 1744-1754.

(26) Reshetnyak, Y. K., Koshevnik, Y., and Burstein, E. A. (2001) Decomposition of protein tryptophan fluorescence spectra into log-normal components. III. Correlation between fluorescence and microenvironment parameters of individual tryptophan residues. Biophys. J. 81, 1735-1758.

(27) Burstein, E. A., Vedenkina, N. S., and Ivkova, M. N. (1973) Fluorescence and the location of tryptophan residues in protein molecules. Photochem. Photobiol. 18, 263-79.

(28) Engelborghs, Y. (2001) The analysis of time resolved protein fluorescence in multi-tryptophan proteins. Spectrochim. Acta. A. Mol. Biomol. Spectrosc. 57, 2255-2270. 